\title{
Ptychographic Complex Imaging Reflectometry for Spatially-Resolved Dopant Profiling Using a Tabletop EUV Source
}

Yuka Esashi $^{1 *}$, Christina L. Porter ${ }^{1}$, Michael Tanksalvala ${ }^{1}$, Galen P. Miley ${ }^{3}$, Naoto Horiguchi ${ }^{4}$, Joshua L. Knobloch ${ }^{1}$, Jihan Zhou ${ }^{5}$, Robert M. Karl Jr. ${ }^{1}$, Peter Johnsen ${ }^{1}$, Charles S. Bevis ${ }^{1}$, Bin Wang ${ }^{1}$, Seth L. Cousin $^{2}$, Michael Gerrity ${ }^{1}$, Henry C. Kapteyn ${ }^{1,2}$, Margaret M. Murnane ${ }^{1,2}$

1. JILA, University of Colorado, Boulder, Colorado 80309, USA.

2. KMLabs, Inc., Boulder, Colorado, 80301, USA.

3. Department of Chemistry, Northwestern University, Evanston, Illinois 60208, USA.

4. imec Leuven, Kapeldreef 75, 3001 Leuven, Belgium.

5. Department of Physics \& Astronomy and California NanoSystem Institute, University of California, Los Angeles, California 90095, USA.

* Corresponding author: yuka.esashi@colorado.edu

Next-generation semiconductor, quantum, and spintronics devices are becoming more complex, as the demand for smaller features necessitates three-dimensional architectures. In order to characterize these nanostructures, there is a growing need for quantitative, high-resolution, and non-destructive metrology techniques of surfaces, interfaces, layers, as well as depth-dependent composition and dopant levels. We present a solution to this challenge: an extreme ultraviolet (EUV) complex imaging reflectometer, which uses EUV light from a table-top high harmonic generation source to image the spatially-dependent complex reflectivity of a sample at many incident angles. By mapping the reflectivity versus incident angle for different regions of the sample, it is possible to determine quantities such as thin film layer thicknesses, chemical composition, interfacial region, and dopant profiles. We expect this new tool to have applicability in a broad range of material science and technology.

Imaging using EUV and X-ray light has unique and powerful capabilities: by combining coherent EUV sources with coherent diffraction imaging, it is now possible to image with high transverse and axial resolution - essentially achieving diffraction-limited imaging at short wavelengths for the first time [1]. Ptychography is a powerful implementation of CDI, where a scanning beam and the sample are deconvolved using the data redundancy that arises from spatially overlapping the scan positions [2]. Moreover, EUV light penetrates materials that are opaque to visible light, making it possible to image buried structures and interfaces [3-6]. In addition, the EUV complex reflectivity of a sample is very sensitive to chemical composition, making this wavelength regime particularly suited for reflectometry applications.

Using our EUV imaging reflectometer shown schematically in Fig. 1, we ptychographically imaged a modified semiconductor industry test sample with spatially varying surface structures and dopant profiles, at many incident angles. The microscope was illuminated by coherent EUV beams at a wavelength of $30 \mathrm{~nm}$ from a table-top high harmonic generation source (a modified KMLabs XUUS). An example reconstructed images at an incidence angle of 25 from grazing is shown in Fig. 1(c). Ptychography reconstructs amplitude and phase of the object, which gives valuable information for reflectometry since phase is often more sensitive to surface topography and chemical composition than the absolute reflectivity. A genetic algorithm was used to solve for the structure thicknesses, interface quality, and depth-resolved chemical composition of the sample that match the reflectance versus incident angle curves from several parts of the sample. The reconstructed depth-dependent chemical composition is shown in Fig. 1(d). The solved-for parameters generally agreed with the sample design parameters and measurements made by an AFM, SIMS, HAADF-STEM and EDS. Notably, it was 
necessary to use multiple measurement techniques to confirm all the variables that our model could simultaneously solve for [7].
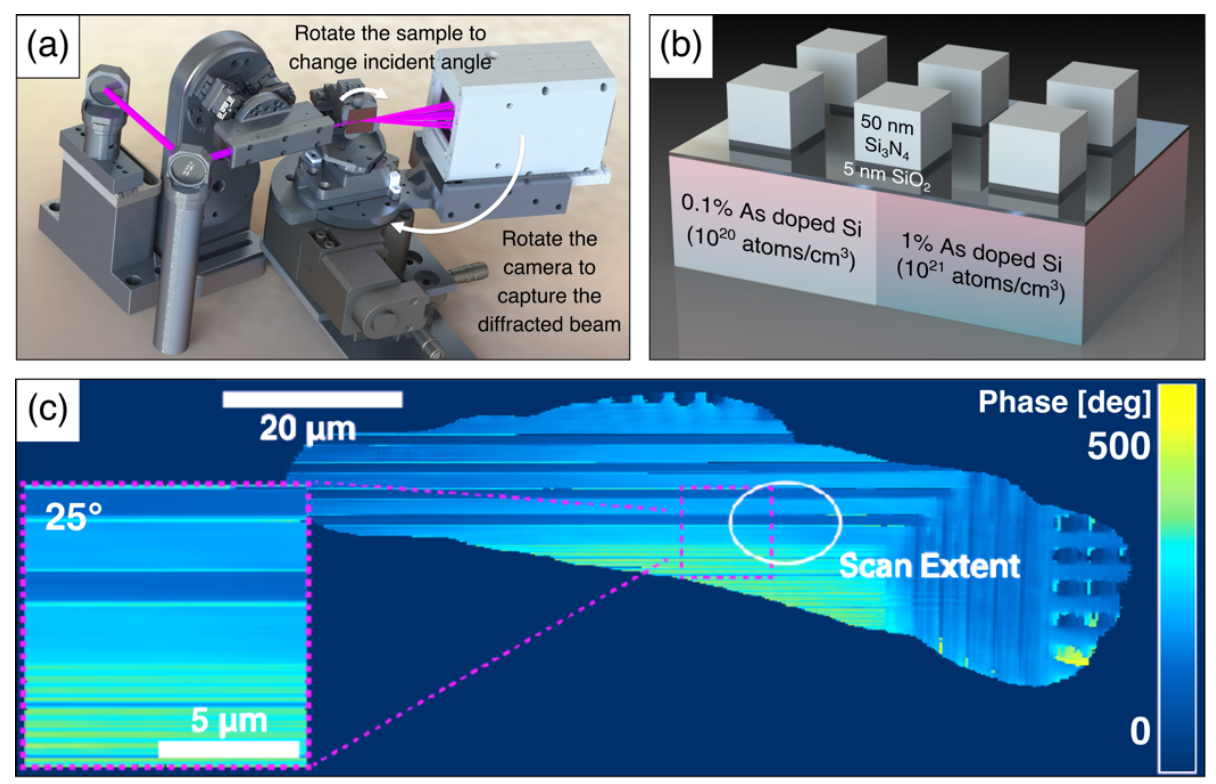

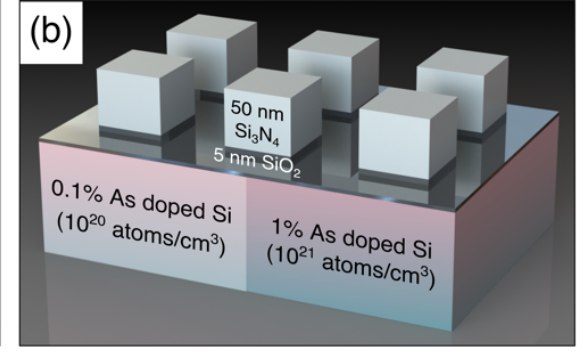

Phase [deg]

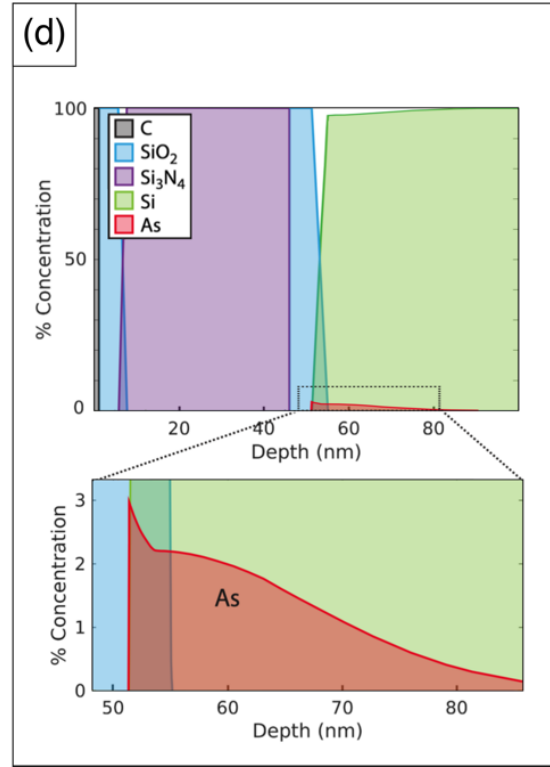

Figure 1. EUV complex imaging reflectometery experiment. (a) The experimental setup schematic. (b) The sample, with $\mathrm{Si}_{3} \mathrm{~N}_{4} / \mathrm{SiO}_{2}$ structures atop $\mathrm{Si}$ substrate, with spatially varying degrees of As doping. (c) Ptychographic phase image of the sample at $25^{\circ}$ from grazing; inset showing the high-fidelity region near the scan extent. (d) Reconstructed depth-dependent chemical composition of a structure on a highly doped region.

\section{References:}

[1] Miao, J., et al, Science 348 (2015), p. 530-535.

[2] Maiden, A. M. and Rodenburg, J. M., Ultramicroscopy 109 (2009), p. 1256-1262.

[3] Shanblatt, E. R., et al, Nano letters 16 (2016), p. 5444-5450.

[4] Gardner, D. F., et al, Nature Photonics 11 (2017), p. 259-263.

[5] Karl, R. M., et al, Science Advances 4 (2018), eaau4295.

[6] Porter, C.L., et al, Optica 4 (2017), p. 1552-1557.

[7] The authors gratefully acknowledge support from NSF STROBE DMR- 1548924, DARPA STTR Award \# W31P4Q-17-C-0104, a Gordon and Betty Moore Foundation EPiQS Initiative through Grant GBMF4538, an NSF GRFP fellowship, and a NDSEG fellowship. H.K. and M.M. are partial owners of KMLabs. 